\title{
Disseminated mucormycosis with cerebellum involvement due to Rhizomucor pusillus in a patient with multiple myeloma and secondary myelodysplastic syndrome: A case report
}

\author{
QIONG CHEN $^{1}$, KUANG CHEN $^{2}$, SHENXIAN QIAN $^{2}$, SHENGHAI WU $^{2}$, LIHUI XU $^{1}$, \\ XILIAN HUANG ${ }^{2}$, PENGFEI SHI ${ }^{2}$, KAILE WANG $^{2}$, MINMIN WANG $^{1}$ and XIANJUN WANG ${ }^{1}$ \\ Departments of ${ }^{1}$ Laboratory Medicine and ${ }^{2}$ Hematology, Affiliated Hangzhou First People's Hospital, \\ Zhejiang University School of Medicine, Hangzhou, Zhejiang 310006, P.R. China
}

Received January 11, 2019; Accepted September 4, 2019

DOI: $10.3892 /$ etm.2019.8065

\begin{abstract}
Mucormycosis is an angioinvasive fungal infection with a high mortality rate. Patients with hematological malignancies following voriconazole therapy are at high risk from mucormycosis. Here, the present study reports on a 68-year-old man diagnosed with multiple myeloma and secondary myelodysplastic syndrome, who was infected with disseminated mucormycosis with cerebellum involvement confirmed by mycological culture and histopathological examination. For patients with hematological malignancies who are receiving antifungal therapy, an opportunistic infection of mucormycosis should be considered if a 'breakthrough' infection occurs in the predilection sites (such as the sinuses, lungs, skin, brain and gastrointestinal tract). It is difficult to diagnose mucormycosis because of the limited reliable detection methods, and because mucormycosis often presents with an acute onset and progresses rapidly, particularly in immunocompromised patients. Antifungal therapy with amphotericin B or posaconazole should be started as soon as possible considering the empirical diagnosis.
\end{abstract}

\section{Introduction}

Mucormycosis is a rare, atypical and life-threatening zygomycosis that is mainly caused by Rhizopus species, Mucor species, Cunninghamella bertholletiae and Rhizomucor species (1-4). Mucormycosis often coexists with other underlying conditions that exhibit immunosuppression(1). Due to the characteristics of invasion of blood vessels and thrombosis, mucormysis usually

Correspondence to: Dr Kuang Chen, Department of Hematology, Affiliated Hangzhou First People's Hospital, Zhejiang University School of Medicine, 261 Huansha Road, Hangzhou, Zhejiang 310006, P.R. China

E-mail: 124974406@qq.com

Key words: mucormycosis, Rhizomucor pusillus, hematological malignancies, mycological culture, autopsy, voriconazole exhibits rapid progression $(3,4)$. According to the anatomical localization, infection patterns could include sinus, pulmonary, cutaneous, cerebral, gastrointestinal or kidney-related and generalized disseminated patterns (1,4-6). Rhinocerebral and pulmonary mucormycosis are the most common manifestation that occurs via air spore inhalation (6). Disseminated mucormycosis is mainly present in hematopoietic stem cell transplant recipients, and usually has a high mortality rate with $>90 \%$ due to progressive invasive infection $(1,7-9)$. The present study reports on a case of cerebral disseminated mucormycosis in a patient with multiple myeloma (MM) and secondary myelodysplastic syndrome (MDS), confirmed by mycological culture and histopathological examination.

\section{Case report}

In January 2013, a 68-year-old man visited Hangzhou First People's hospital due to backache and a fever. The initial laboratory tests showed hyperimmunoglobulin with immune globulin (Ig) A at $2.64 \mathrm{~g} / \mathrm{l}$. The 24-h $\kappa$ light chain level was $6,860 \mathrm{mg} / 24 \mathrm{~h}$ and the serum $\beta 2$-microglobulin level was $2,563 \mathrm{mg} / \mathrm{l}$. In addition, the serum albumin level was $38.9 \mathrm{~g} / \mathrm{l}$. Bone marrow smears revealed $13.5 \%$ abnormal plasma cells. MRI determined vertebral compression fractures at T6 and T11. The pathological and flow cytometry results of bone marrow specimens with plasma cell antigens was CD117+CD38+CD138+CD56-cKappa+, and MM was diagnosed as Durie-Salmon stage II phase A and International Staging System stage I phase A.

The patient was administered chemotherapy including vincristine with $0.5 \mathrm{mg}$ per day (from the first day 4), doxorubicin with $10 \mathrm{mg} / \mathrm{m}^{2}$ per day $\left(10 \mathrm{mg} / \mathrm{m}^{2} / \mathrm{d}\right.$ for days $\left.1-4\right)$ and dexamethasone with $40 \mathrm{mg} /$ day (days 1-4) on January 30, 2013. However, he responded poorly after two courses of chemotherapy and refused to accept bortezomib treatment, so CyRd chemotherapy comprising cyclophosphamide with $300 \mathrm{mg}$ per $\mathrm{m}^{2}$ per week (300 mg/m² $/ \mathrm{w}$ for 4 weeks), lenalidomide with $15 \mathrm{mg} /$ day (from days 1-21) and dexamethasone with $40 \mathrm{mg}$ per week (40 mg/w) was administered to him on 16 April, 2013. After six courses of the CyRd regimen, the patient showed a very good partial response. Thereafter, lenalidomide was used 
as maintenance therapy with $15 \mathrm{mg} /$ day; continuous medication for 21 days with an interval of 7 days in a 28-day-course treatment.

In May 2016, the patient was admitted to Affiliated Hangzhou First People's hospital again due to disease progression with fatigue and dizziness. Bone marrow pathology and chromosome karyotyping revealed MM progression and secondary MDS. Therefore, the patient was administered bortezomib (1.3 $\mathrm{mg} / \mathrm{m}^{2} /$ week) combined with dexamethasone (40 mg; qw), but had a poor response. Since the previous chemotherapy treatment, the patient had lost weight and had been diagnosed with diabetes. However, the blood glucose levels of the patient were not well controlled due to incomplete compliance with insulin treatment.

On October 20, 2017, the patient visited the hospital after suffering from chills and fever lasting 1 week. CT showed acute pneumonia in the right middle lung (Fig. 1A and B). The cranial MRI result was normal. The results were negative with etiological separation and culture, T-SPOT test for tuberculosis infection, the galactomannan test for invasive pulmonary aspergillosis, normal cytomegalovirus DNA and endotoxin levels. The level of 1-3- $\beta$-D glucan was $550.40 \mathrm{ng} / \mathrm{l}$. Due to the antibiotic agents having minimal effect, voriconazole (200 mg; twice per day) was empirically prescribed for antifungal treatment in considering probable invasive aspergillosis. The infection seemed to be controlled, as C-reactive protein (CRP) level and body temperature were normal. On November 24, 2017, due to the progressive MM with a level of $24-\mathrm{h} \kappa$ light at $19,548 \mathrm{mg} / 24 \mathrm{~h}$, the patient underwent chemotherapy with reduced doses of bortezomib $\left(1.1 \mathrm{mg} / \mathrm{m}^{2} /\right.$ week $)$, cyclophosphamide (300 mg; qw) and dexamethasone (20 mg; qw) due to his low weight, and the level of 24-h urine $\kappa$ light chain was significantly reduced to $2543 \mathrm{mg} / 24 \mathrm{~h}$. Thereafter, the patient underwent outpatient follow-up treatment.

During the period of outpatient follow-up treatment, the patient independently reduced the oral dose of voriconazole due to incomplete compliance. A period of 1 week later, fever presented again with a dry cough. On December 19, 2017, the patient was readmitted to Hangzhou First People's Hospital. The previous infection in the right middle lung was improved according to pulmonary CT images, but new infection sites had appeared in the right lower lung (Fig. 1C and D). MRI displayed certain abnormal signal lesions with new infection in the right cerebellum (Fig. 1E-G). At this time, combined therapy of voriconazole (200 $\mathrm{mg}$, twice daily), meropenem ( $2.0 \mathrm{~g}$, three times daily) and linezolid (0.6 g, twice daily) was started for the patient, but it was ineffective. After 10 days, the patient lost consciousness and was considered to exhibit secondary occipital foramen with high intracranial pressure. Immediately, emergency surgery was performed for decompression, showing high tension in the cerebellum, and the operation was conservative with an extremely low platelet count $\left(18 \times 10^{9} / 1\right)$. The brain tissue showed necrosis and was obtained for histopathological and etiological examination. That night, the patient was in a persistent coma, and subsequently died due to myocardial infarction with ST-segment arch-back elevation in the multi-lead anterior intermediate wall (cardiac troponin I, $7.8 \mathrm{ng} / \mathrm{ml}$; creatine kinase-MB, $57 \mathrm{ng} / \mathrm{ml}$ ). The patient's laboratory tests and basic clinical data are presented in Table SI.
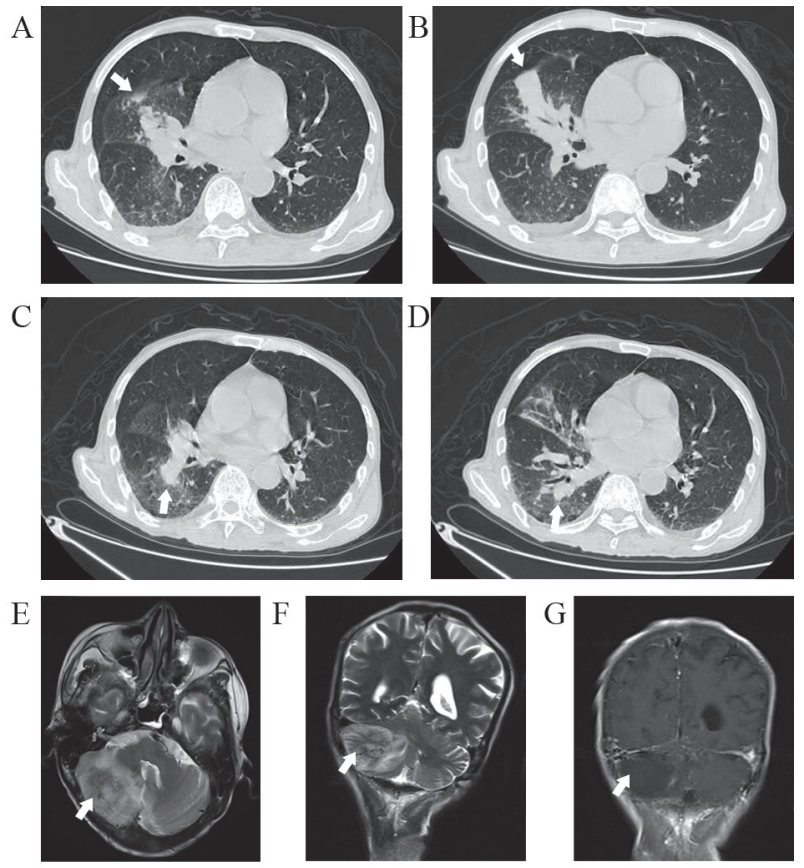

Figure 1.Imaging findings.(A) Pulmonary CT scans showing acute pneumonia in the right middle lung (23.10.2017). (B) Pulmonary CT scans showing acute pneumonia in the right middle lung (23.10.2017). (C) Pulmonary CT scans for mucormycosis in the right lower lung (December 25, 2017). (D) Pulmonary CT scans for mucormycosis in the right lower lung (December 25, 2017). (E) MRI of the cerebellum (December 28, 2017). (F) MRI of the cerebellum (December 28, 2017). (G) MRI of the cerebellum (December 28, 2017).

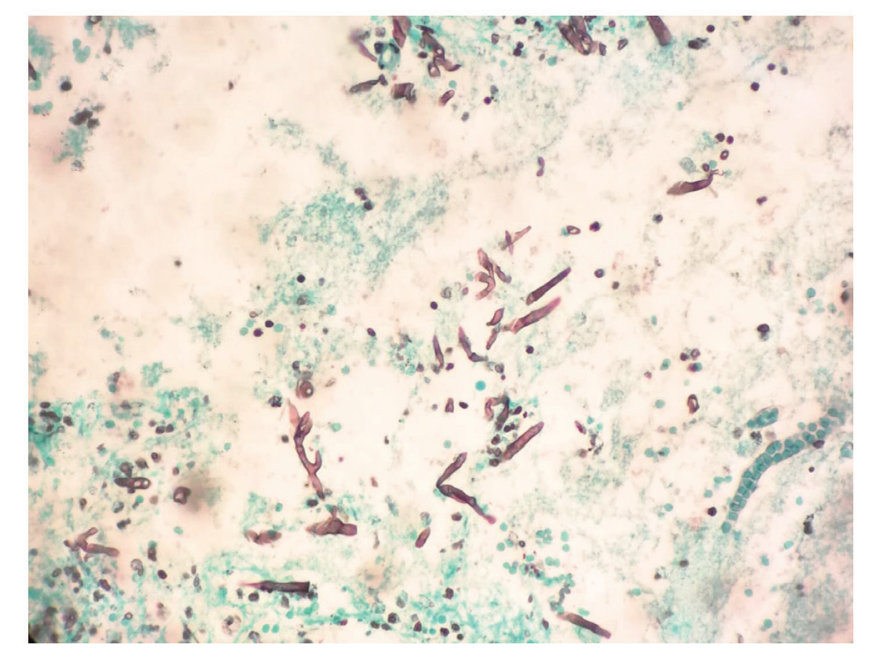

Figure 2. Histological analysis of a brain sample showing broad-based non-septate hyphae consistent with Mucor species following silver staining. Magnification, $\mathrm{x} 400$.

Histopathology demonstrated neutrophil infiltration and large broad-based non-septate hyphae upon Gomori methenamine silver staining, leading to a diagnosis of Mucor species (Fig. 2). Mycological cultures were positive in three days with a loose filamentous fungal colony. Both the colonial and microscopic features suggested Mucor species (Fig. 3). The antifungal susceptibility test was performed using the broth microdilution method as previously documented $(10,11)$, and the results showed that the minimum inhibitory concentrations of posaconazole and amphotericin B were 0.25 and $1 \mathrm{mg} / \mathrm{l}$, 

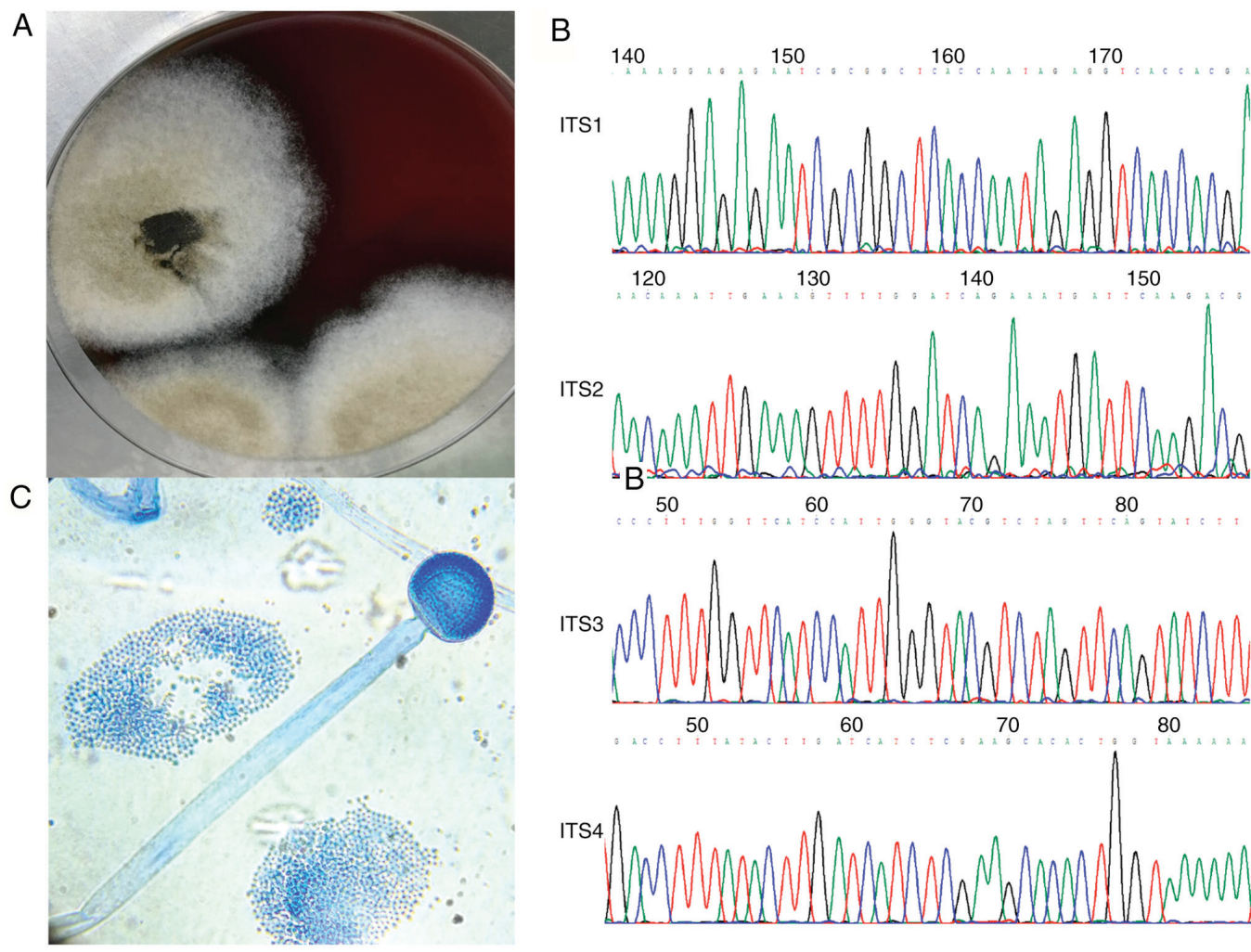

Figure 3. Colonial and microscopic feature of the fungus, and ITS region sequencing. (A) Rhizomucor pusillus in blood agar. (B) ITS sequence peak maps. (C) Lactophenol cotton blue mount preparations. Magnification, x400. ITS, internal transcribed spacer.

respectively. However, there was resistance to voriconazole and itraconazole, according to the breakpoint of Aspergillus (Table I). Internal transcribed spacer region sequencing confirmed the pathogenic basis of Rhizomucor pusillus (Genbank numbers: MN061000 and MN061001in the database of the National Center for Biotechnology Information; Fig. 3).

\section{Discussion}

In the present study, due to primary hematological malignancy and long-term use of chemotherapy drugs, the patient had additional high-risk factors for invasive fungal infections, including diabetes, poor immunity and immunosuppression $(4,6)$. The patient likely contracted two different invasive fungal infections in a short time. On October 20,2017, the patient may have been infected with a voriconazole-susceptible fungus without etiological support, because the right middle lung imaging manifestations were improved following voriconazole treatment, with the patient displaying normal CRP levels and normal body temperature. On December 19, 2017, the patient was readmitted to the hospital with a probable new fungal infection that was secondary to voriconazole treatment and was non-susceptible to voriconazole. However, when the new infection site appeared in the right lower lung and brain, the doctor incorrectly assumed the cause to be a secondary infection, in addition to the first fungal infection, and failed to identify a possible new Mucor infection. The present study concluded that if the doctor had noticed the Mucor infection, and if the patient had been appropriately treated with posaconazole, the patient may have been cured of this infection.
Although it is difficult to identify the etiological diagnosis for patients with high-risk factors due to the limited diagnostic methods $(12,13)$ for a clinical presentation of invasive fungal infection, it is helpful to use antifungal therapy appropriately as an empirical treatment. The current report revealed that voriconazole treatment may increase the risk of Mucor infection within a short time in high-risk patients. Previous studies determined that using voriconazole, especially in patients with hematological malignancy and recipients of hematopoietic stem cell transplants, is associated with an increased incidence of mucormycosis $(5,14)$. Mucormycosis infection can occur as a breakthrough infection during voriconazole treatment (15). The patient presented in the present case report might have had a breakthrough infection following treatment with voriconazole.

Mucormycosis is an aggressive and angioinvasive fungal infection responsible for high morbidity and mortality $(6,13)$. Although the number of invasive mucormycosis cases has increased in recent years due to the increased prevalence of immunosuppression in the general population $(7,16,17)$, it remains an uncommon infection $(5,18)$. Invasive mucormycosis often occurs in high-risk populations, such as those with diabetes mellitus, hematological malignancies and other immunocompromised states $(1,4,19,20)$. Even in immunocompetent populations, such as those with trauma, burns or undergoing surgery, invasive mucormycosis may occur, as documented in previous reports $(7,12,18,19)$. In the present report, a patient with MM and secondary MDS demonstrated disseminated mucormycosis with cerebral involvement during voriconazole treatment. For high-risk patients who undergo voriconazole therapy, mucormycosis infection should be considered a possibility. 
Table I. Minimum inhibitory concentrations of different antifungal agents.

\begin{tabular}{|c|c|c|c|c|}
\hline \multirow[b]{2}{*}{ Antifungal agents ${ }^{\mathrm{a}}$} & \multirow[b]{2}{*}{$\mathrm{MIC}, \mathrm{mg} / \mathrm{l}$} & \multicolumn{3}{|c|}{ Breakpoint } \\
\hline & & Susceptible & Intermediate & Resistant \\
\hline Amphotericin B & 1 & $\leq 1$ & 2 & $\geq 4$ \\
\hline Itraconazole & $>256$ & $\leq 1$ & 2 & $\geq 4$ \\
\hline Posaconazole & 0.25 & $\leq 1$ & 2 & $\geq 4$ \\
\hline Voriconazole & $>256$ & $\leq 1$ & 2 & $\geq 4$ \\
\hline Anidulafungin & $>8$ & - & - & - \\
\hline Micafungin & $>8$ & - & - & - \\
\hline Caspofungin & $>8$ & - & - & - \\
\hline 5-Fluorocytosine & $>64$ & - & - & - \\
\hline
\end{tabular}

${ }^{a}$ The interpretive categories and MIC breakpoints are in reference to Aspergillus, according to clinical and laboratory standards institute M38 edition (CLSI M38) (11). -, no interpretive categories; MIC, minimum inhibitory concentration.

Due to the angioinvasive ability of mucormycosis, it can readily cause necrosis and thrombosis (21). The embolus spreads and is implanted into other sites, and may develop into disseminated mucormycosis. The central nervous system (CNS) is a common dissemination site $(1,3)$, and the rhino-orbito-cerebral type is the predominant infection pattern $(3,4)$. Disseminated mucormycosis is a life-threatening disease with a high mortality rate. Among 174 renal transplant recipients with mucormycosis, disseminated patterns accounted for $14.4 \%$ of cases and the mortality rate was $76.0 \%$, compared with the overall mortality rate of $42.5 \%$ (22). In a retrospective investigation, 929 patients were diagnosed with zygomycosis, and 283 had CNS involvement with a mortality rate of $62 \%$, with all 25 patients with generalized disseminated infection succumbing to the disease (7). In contrast to the more common rhino-orbito-cerebral mucormycosis, the infection site in the patient presented here was rare, as it occurred in the cerebellum. Disseminated cerebellar mucormycosis may have spread from the lung to the brain through the blood circulation.

To date, no common reliable clinical serological diagnostic method has been developed for mucormycosis $(13,20)$. Histopathological examination seems to be the gold standard, as this method is able to the identify characteristic of non-septate hyphae branching at a right angle. Autopsy is another reliable invasive technique for tissue samples; however, the diagnosis rates are low, even in high-risk patients $(1,6)$. Microbial culture is a time-consuming method with low sensitivity (17). Additionally, DNA sequencing targeting the region directly from tissue has provided a promising method for pathogenic detection (23), but sequencing is challenging in a basic laboratory setting. In most cases, high-risk patients with mucormycosis are empirically treated with antifungal therapy without histopathological and microbiological support. Thus, the diagnosis of fungal infection not only depends on the empirical work of the doctor, but is also supported by effective testing technology in the laboratory (21).

Regarding mucormycosis therapy, amphotericin B and amphotericin B lipid formulation are the most common antifungal agents for mucormycosis $(5,18)$. Posaconazole is a novel azole antifungal for treating mucormycosis $(5,24)$. A previous study demonstrated that antifungal treatments combined with surgical debridement are important therapies for mucormycosis $(17,25)$.

In conclusion, the present study described disseminated mucormycosis in a patient with MM and secondary MDS. Mucormycosis can be considered a breakthrough infection in immunosuppressed patients who are receiving voriconazole therapy. In addition to early diagnosis, appropriate initiation of chemotherapy and surgical debridement are important.

\section{Acknowledgements}

The authors would like to thank Dr Panpan Zhao from the Pathology Department in the Hangzhou First People's Hospital for providing the silver stain picture.

\section{Funding}

This work was supported by National Natural Science Foundation of China (grant no. 81601799), and Hangzhou Science and Technology Commission Social Development Project (grant no. 20180533B31).

\section{Availability of data and materials}

All data generated or analyzed during the present study are included in this published article and its supplementary information files.

\section{Authors' contributions}

QC and KC contributed to study design, clinical information collection, data interpretation, literature search and manuscript preparation. SXQ, XLH, PFS and KLW contributed to the collection of clinical information, the literature search and case analysis. SHW, LHX, MMW and XJW performed to the laboratory examinations, the results analysis and interpretation. All authors read and approved the final version of the manuscript. 


\section{Ethics approval and consent to participate}

Informed consent was obtained from the patient.

\section{Patient consent for publication}

Informed consent for publication was obtained from the son of the patient, due to the patient passing away.

\section{Competing interests}

The authors declare that they have no competing interests.

\section{References}

1. Petrikkos G, Skiada A, Lortholary O, Roilides E, Walsh TJ and Kontoyiannis DP: Epidemiology and clinical manifestations of mucormycosis. Clin Infect Dis 54 (Suppl 1): S23-S34, 2012.

2. Kauffman CA: Zygomycosis: Reemergence of an old pathogen. Clin Infect Dis 39: 588-590, 2004.

3. Vaezi A, Moazeni M, Rahimi MT, de Hoog S and Badali H: Mucormycosis in Iran: A systematic review. Mycoses 59: 402-415, 2016

4. Jeong W, Keighley C, Wolfe R, Lee WL, Slavin MA, Kong DCM and Chen SC: The epidemiology and clinical manifestations of mucormycosis: A systematic review and meta-analysis of case reports. Clin Microbiol Infect 25: 26-34, 2019.

5. Kursun E, Turunc T, Demiroglu YZ, Aliskan HE and Arslan AH: Evaluation of 28 cases of mucormycosis. Mycoses 58: 82-87, 2015.

6. Serris A, Danion F and Lanternier F: Disease entities in mucormycosis. J Fungi (Basel) 5: pii: E23, 2019.

7. Roden MM, Zaoutis TE, Buchanan WL, Knudsen TA, Sarkisova TA, Schaufele RL, Sein M, Sein T, Chiou CC, $\mathrm{Chu} \mathrm{JH}$, et al: Epidemiology and outcome of zygomycosis: A review of 929 reported cases. Clin Infect Dis 41: 634-653, 2005.

8. Andrey DO, Kaiser L, Emonet S, Erard V, Chalandon Y and van Delden C: Cerebral Rhizomucor infection treated by Posaconazole delayed-release tablets in an allogeneic stem cell transplant recipient. Int J Infect Dis 55: 24-26, 2017.

9. Xipell M, Losno RA, Garcia-Vidal C, Rovira M, Alejo-Cancho I, Puig de la Bellacasa J, López M, Cardozo C, Bodro M, Mensa J and Soriano A: Clinical features and outcome in patients with mucormycosis in a tertiary hospital (2012-2016). Rev Iberoam Micol 35: 162-166, 2018 (In Spanish).

10. Posteraro B, Spanu T, Fiori B, De Maio F, De Carolis E, Giaquinto A, Prete V, De Angelis G, Torelli R, D'Inzeo T, et al: Antifungal susceptibility profiles of bloodstream yeast isolates by Sensititre YeastOne over nine years at a large Italian teaching hospital. Antimicrob Agents Chemother 59: 3944-3955, 2015.

11. Clinical and Laboratory Standards Institute (CLSI): M38. Reference method for broth dilution antifungal susceptibility testing of filamentous Fungi, 3rd edition. CLSI, Wayne, PA, 2017. https://clsi.org/standards/products/microbiology/documents/m38/. Accessed November 30, 2017.
12. Kennedy KJ, Daveson K, Slavin MA, van Hal SJ, Sorrell TC, Lee A, Marriott DJ, Chapman B, Halliday CL, Hajkowicz K, et al: Mucormycosis in Australia: Contemporary epidemiology and outcomes. Clin Microbiol Infect 22: 775-781, 2016.

13. Long B and Koyfman A: Mucormycosis: What emergency physicians need to know? Am J Emerg Med 33: 1823-1825, 2015.

14. Ville S, Talarmin JP, Gaultier-Lintia A, Bouquié R, Sagan C, Le Pape P, Giral M and Morio F: Disseminated mucormycosis with cerebral involvement owing to rhizopus microsporus in a kidney recipient treated with combined liposomal amphotericin B and posaconazole therapy. Exp Clin Transplant 14: 96-99, 2016.

15. Chamilos G, Marom EM, Lewis RE, Lionakis MS and Kontoyiannis DP: Predictors of pulmonary zygomycosis versus invasive pulmonary aspergillosis in patients with cancer. Clin Infect Dis 41: 60-66, 2005.

16. Bitar D, Van Cauteren D, Lanternier F, Dannaoui E, Che D, Dromer F, Desenclos JC and Lortholary O: Increasing incidence of zygomycosis (mucormycosis), France, 1997-2006. Emerg Infect Dis 15: 1395-1401, 2009.

17. Cornely OA, Arikan-Akdagli S, Dannaoui E, Groll AH, Lagrou K, Chakrabarti A, Lanternier F, Pagano L, Skiada A, Akova M, et al: ESCMID and ECMM joint clinical guidelines for the diagnosis and management of mucormycosis 2013. Clin Microbiol Infect 20 (Suppl 3): S5-S26, 2014.

18. Lin E, Moua T and Limper AH: Pulmonary mucormycosis: Clinical features and outcomes. Infection 45: 443-448, 2017.

19. Wang Q, Liu B and Yan Y: Disseminated mucormycosis (DM) after pneumonectomy: A case report. BMC Infect Dis 16: 337, 2016.

20. Walsh TJ, Gamaletsou MN, McGinnis MR, Hayden RT and Kontoyiannis DP: Early clinical and laboratory diagnosis of invasive pulmonary, extrapulmonary and disseminated mucormycosis (zygomycosis). Clin Infect Dis 54 (Suppl 1): S55-S60, 2012.

21. Paul SR and Gable PS: Mucormycosis as the elusive cause of an aortic thrombus and tissue-obliterating abscess. Case Rep Hematol 2019: 4842150, 2019.

22. Song Y, Qiao J, Giovanni G, Liu G, Yang H, Wu J and Chen J: Mucormycosis in renal transplant recipients: Review of 174 reported cases. BMC Infect Dis 17: 283, 2017.

23. Iwen PC, Freifeld AG, Sigler L and Tarantolo SR: Molecular identification of Rhizomucor pusillus as a cause of sinus-orbital zygomycosis in a patient with acute myelogenous leukemia. J Clin Microbiol 43: 5819-5821, 2005.

24. Dannaoui E, Meletiadis J, Mouton JW, Meis JF and Verweij PE; Eurofung Network: In vitro susceptibilities of zygomycetes to conventional and new antifungals. J Antimicrob Chemother 51: 45-52, 2003.

25. Multani A, Reveron-Thornton R, Garvert DW, Gomez CA, Montoya JG and Lui NS: Cut it out! Thoracic Surgeon's approach to pulmonary mucormycosis and the role of surgical resection in survival. Mycoses 62: 893-907, 2019.

This work is licensed under a Creative Commons Attribution-NonCommercial-NoDerivatives 4.0 International (CC BY-NC-ND 4.0) License. 